\title{
THEORETICAL INVESTIGATIONS ON STANDING WAVE THERMOACOUSTIC PRIME MOVER USING DELTAEC
}

\author{
Ajinkya Sarode ${ }^{1}$, Vijay Dighe ${ }^{2}$, Vinay Ingle ${ }^{3}$, Nilesh Kolekar ${ }^{4}$ \\ ${ }^{l}$ M.Tech Student, Mechanical Engineering Department, Sardar Vallabhbhai National Institute of Technology (SVNIT), \\ Surat, Gujarat, India \\ ${ }^{2}$ M.Tech Student, Mechanical Engineering Department, Sardar Vallabhbhai National Institute of Technology (SVNIT), \\ Surat, Gujarat, India \\ ${ }^{3}$ M.Tech Student, Mechanical Engineering Department, Sardar Vallabhbhai National Institute of Technology (SVNIT), \\ Surat, Gujarat, India \\ ${ }^{4}$ M.Tech Student, Mechanical Engineering Department, Sardar Vallabhbhai National Institute of Technology (SVNIT), \\ Surat, Gujarat, India
}

\begin{abstract}
Thermoacoustic is a field of science and technology that studies heat and sound interactions. It deals with the conversion of thermal energy into acoustical energy and vice-versa. Thermoacoustic prime mover is an attractive alternative for a conventional pressure wave generator to drive cryocooler which attains cryogenic temperature below $123 \mathrm{~K}$ to liquefy gases, to produce ultra-vacuum, etc. Also, it can be used to produce electricity with the help of thermoacoustic electric generator. The cooling effect generated by thermoacoustic refrigerator can be used in areas where there is no supply of electricity. The advantages of thermoacoustic prime mover lie in its less complicated construction, absence of any moving part and use of environmental friendly gases as a working medium. Number of simulations using gases such as $\mathrm{He}, \mathrm{Ar}, \mathrm{N}_{2}$ and $\mathrm{He}-\mathrm{Ar}$ mixture were carried out in DeltaEC to investigate the performance of the prime mover in terms of pressure amplitude, onset temperature and resonating frequency. Input parameters like working pressure and heat input was varied to observe the effect on the performance of the prime mover. The results showed that as the working pressure increased, the pressure amplitude and onset temperature also increased. Similar effect was observed in case of heat input. Unlike pressure amplitude and onset temperature, resonating frequency does not depend on working pressure and heat input. It is controlled by the working gas and the geometry of the prime mover. Also the type of the working gas has a profound impact on the performance of the prime mover. Simulations also demonstrated that a mixture of He-Ar can be used to get the optimum output from the prime mover.
\end{abstract}

Keywords: Thermoacoustic prime mover, onset temperature, pressure amplitude, resonating frequency, DeltaEC.

\section{INTRODUCTION}

The thermodynamic interaction between acoustics and solid surfaces that possess a temperature gradient is termed as thermoacoustic effect. Recent years has witnessed a rapid developed in thermoacoustic technology. Devices such as thermoacoustic engine, thermoacoustic refrigerator and thermo acoustically driven pulse tube cryocoolers are the outcomes of this field. Thermoacoustic engines convert thermal energy into acoustic power in the form of high amplitude sound waves. The heat transfer from the working fluid to stack and vice versa gives rise to thermoacoustic oscillations. This acoustic power is used to drive thermoacoustic pulse tube cryocooler thermoacoustic refrigerators and thermoacoustic electric generators. Eradication of mechanical pistons such as compressors, use of environment friendly gases as working medium and no maintenance are the important reasons for the increasing interest in thermoacoustics. The performance of the standing wave thermoacoustic engine depends on number of parameters like working fluid, heat input, working pressure, geometry of the prime mover, etc. Gases such as helium (He), nitrogen $\left(\mathrm{N}_{2}\right)$, argon $(\mathrm{Ar})$, carbon-dioxide $\left(\mathrm{CO}_{2}\right)$ and their mixtures are used as the working fluid in the thermoacoustic prime mover. The aim of this report is to theoretically investigate the effect of working pressure, working fluid and heat input through simulation using DeltaEC software. 


\begin{tabular}{|llll|}
\hline \multicolumn{2}{|l|}{ Nomenclature } & & \\
$B R$ & blockage ratio & $H$ & normalised total power flux through stack $(\mathrm{W})$ \\
$y_{0}$ & half of plate spacing $(\mathrm{m})$ & $\gamma$ & ratio of isobaric to isochoric specific heat \\
$l$ & half of plate thickness $(\mathrm{m})$ & $R$ & inner radius of resonator pipe $(\mathrm{m})$ \\
$\delta_{k}$ & thermal penetration depth $(\mathrm{m})$ & $\Gamma$ & normalised temperature gradient \\
$k$ & thermal conductivity $(\mathrm{W} / \mathrm{mK})$ & $\sigma$ & Prandtl number \\
$\omega$ & angular frequency $(\mathrm{radian})$ & $\delta_{k n}$ & normalised thermal penetration depth \\
$\rho$ & density of gas $\left(\mathrm{kg} / \mathrm{m}^{3}\right)$ & $x_{n}$ & normalised stack centre position \\
$c_{p}$ & specific heat of gas $(\mathrm{J} / \mathrm{kgk})$ & $\delta_{v n}$ & normalised viscous penetration depth \\
$\eta_{t h}$ & thermal efficiency & $\Delta T_{n}$ & normalised temperature difference \\
$\Delta E$ & acoustic power generated from the $\operatorname{stack}(\mathrm{W})$ & $a$ & acoustic velocity $(\mathrm{m} / \mathrm{s})$ \\
\hline
\end{tabular}

\section{SIMULATION IN DELTAEC}

DeltaEC (Design Environment for Low Amplitude Thermoacoustic Energy Conversion) developed by Ward and Swift is used to investigate the performance of the thermoacoustic prime mover. The prime mover is simulated in DeltaEC and the performance is evaluated using various gases at different pressures and heat input. The half wave standing thermoacoustic prime mover consists of parts such as hot heat exchanger, cold heat exchanger, stack, resonator and working gas. as shown in the schematic diagram Figure 1 [1].

\subsection{Heat Exchangers}

The hot and cold heat exchanger plays an important role in maintaining a temperature gradient across the stack of the prime mover. The hot heat exchanger supplies heat to the working fluid and the cold heat exchanger draws the heat from the working fluid. The heat transfer coefficient should be high to provide efficient heat transfer. The porosity of the cold heat exchanger and the stack should be same to avoid entrance losses of gas parcels.

Parallel plate type heat exchangers made of copper and having a porosity of 0.4 is used for hot heat exchanger and 0.6 for cold heat exchanger in the simulation. To ensure effective heat transfer from hot heat exchanger to the working gas, its porosity is kept low. The porosity or blockage ratio (BR) is defined as the ratio of area through which the gas is passing to the total area of the heat exchanger. The porosity of any heat exchanger is given by the following equation,

$$
B R=\frac{y_{0}}{y_{0}+l}
$$

Where, $y_{0}$ and $l$ are half of the plate spacing and plate thickness respectively [2].

\subsection{Stack}

Stack is the most important component of the prime mover as it is the source of the thermoacoustic oscillations. It should have high heat capacity to maintain constant temperature gradient and low thermal conductivity to avoid axial heat conduction from hot heat exchanger to cold heat exchanger. The position of the stack in the prime mover should be such that the magnitude of gas velocity amplitude should be low to reduce the viscous losses. The minimum plate spacing of the stack depends on thermal penetration depth $\left(\delta_{k}\right)$ and it is generally kept as 2-3 times of $\delta_{k}$ to have imperfect thermal contact between gas and plate in order to have phase difference in case of standing wave thermoacoustic prime mover. Thermal penetration depth is defined as the distance through which the diffusion of heat can take place to the gas. In the simulation, stack made of stainless steel is used which is a parallel plate type having BR same as that of the cold heat exchanger.

The expression for the thermal penetration depth $\left(\delta_{k}\right)$ is given by,

$$
\delta_{k}=\sqrt{\frac{2 k}{\omega \rho c_{p}}}
$$

Where, $k, \omega, \rho, c_{p}$ are thermal conductivity, angular frequency, density and specific heat at constant pressure of gas, respectively.

The thermal efficiency of the thermoacoustic prime mover is the ratio of normalized acoustic power developed by the engine to the total power supplied to the engine through hot heat exchanger and it is given as, [3]

$$
\eta_{t h}=\frac{\Delta E}{H}
$$

Normalised acoustic power is given by,

$$
\begin{gathered}
\Delta E=\frac{1}{4 \gamma} \delta_{k n} D R^{2}\left[B R(\gamma-1) \cos ^{2}\left(x_{n}\right)\left(\frac{\Gamma}{(1+\sqrt{\sigma}) \Lambda}-1\right)\right. \\
\left.-\frac{\sin ^{2}\left(x_{n}\right) \sqrt{\sigma}}{B R \times \Lambda}\right]
\end{gathered}
$$




$$
\begin{aligned}
& H=\frac{1}{8 \gamma} \delta_{k n} D R^{2 \sin \left(2 x_{n}\right)} \frac{1+\sqrt{\sigma}+\sigma}{(1+\sigma) \Lambda}- \\
& \left.\left(1+\sqrt{\sigma}-\delta_{v n}\right)\right] \\
& \Lambda=1-\sqrt{\sigma} \delta_{k n}+\frac{1}{2} \sigma \delta_{k n}^{2} \\
& \Gamma=\frac{\Delta T_{n}}{B R(\gamma-1) L_{s n} \cot \left(x_{n}\right)}
\end{aligned}
$$

Multiplication of wave number $(\omega / a)$ to the actual stack position and length gives the normalized stack position and length. The ratio of penetration depth to $y_{0}$ gives the normalized penetration depth.

\subsection{Resonator}

A resonant tube is also an important part of an engine as it decides the resonant frequency and velocity and pressure profiles. It is a smooth, linear cylindrical pipe without steps, misalignments and abrupt transitions connected after cold heat exchanger to avoid unwanted eddying or non-linear pressure variations that can greatly complicate the analysis. It is a long pipe where oscillating gas and sound wave are in resonance. The material of the resonator should have high impedance so that it will behave as a rigid boundary and will minimize the viscous losses. Also, to avoid axial heat conduction the material should have less thermal conductivity.
Hence, in simulation resonator made of stainless steel is used. Resonator losses such as thermal and viscous losses are directly proportional to its surface area [4]. Also, if the length of the resonator is too long compared to stack, the prime mover may oscillate in its second node and increase the losses [5]. Thus, in order to minimise the losses, it is preferred to use resonator having less surface area.

\subsection{Working Gas}

The performance of the thermoacoustic prime mover highly depends on the properties of the working gas. Prandtl number which gives the relation between viscous penetration depth to thermal penetration depth plays an important role in evaluating the performance of the engine. Generally, gases having low Prandtl number and high ratio of specific heat are suitable for thermoacoustic engine in order to reduce the viscous losses. In order to optimize the properties of the working fluid, mixtures are used in appropriate compositions. In this simulation, gases such as Helium, Nitrogen, Argon and He-Ar mixture are used to compare the performance of the engine.

Figure 2 shows the model of the thermoacoustic prime mover used during the simulation in DeltaEC.

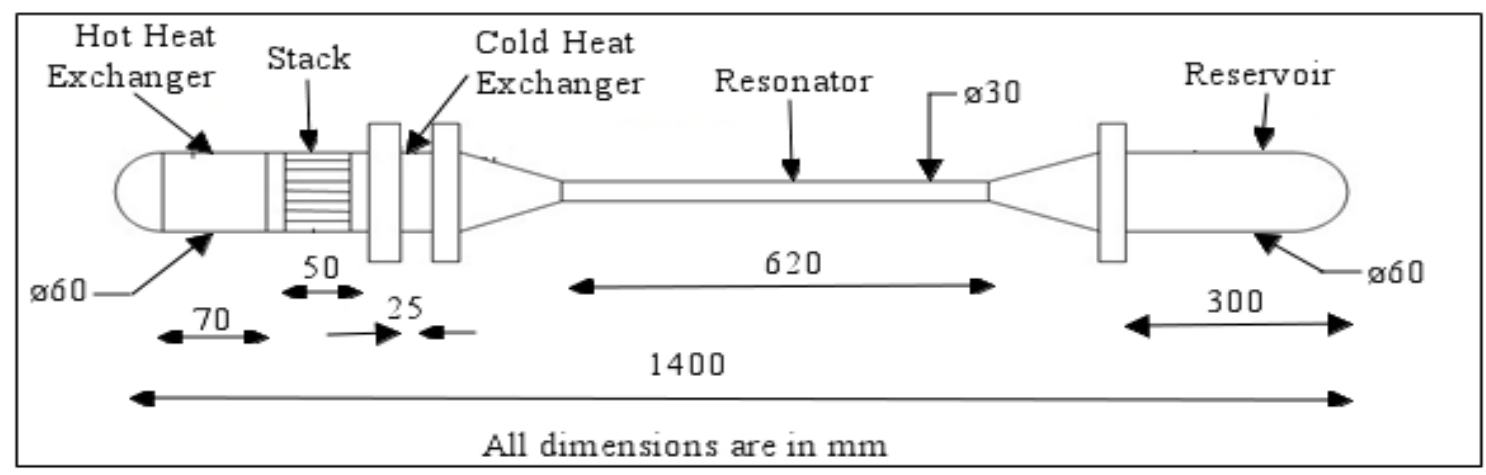

Fig -1: Schematic diagram of Standing wave thermoacoustic prime mover

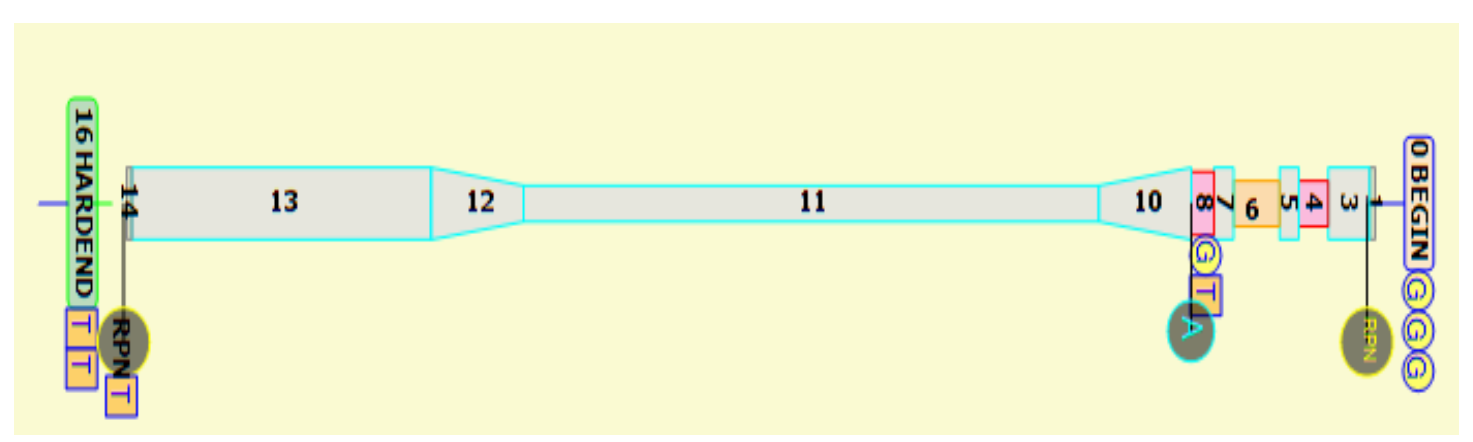

Fig -2: Model of Standing wave thermoacoustic prime mover in DeltaEC 


\section{RESULTS AND DISCUSSIONS}

The model shown in Fig. 2 of the standing wave thermoacoustic prime mover was simulated using DeltaEC. The performance of the engine was evaluated in terms of pressure amplitude, onset temperature and resonant frequency by varying working pressure, working gas and heat input.

\subsection{Effect of Working Pressure}

The variation in working pressure has an effect on the pressure amplitude, onset temperature and resonating frequency of the prime mover. This variation is compared for $\mathrm{He}, \mathrm{N}_{2}, \mathrm{Ar}$ and $\mathrm{He}-\mathrm{Ar}$ mixture. During this simulation, the cold end temperature was maintained to $303 \mathrm{~K}$ and the heat input to the system was kept constant at $1500 \mathrm{~W}$.

\subsubsection{Pressure Amplitude}

As shown in Graph. 1, the pressure amplitude for various gases has been plotted with respect to working pressure.

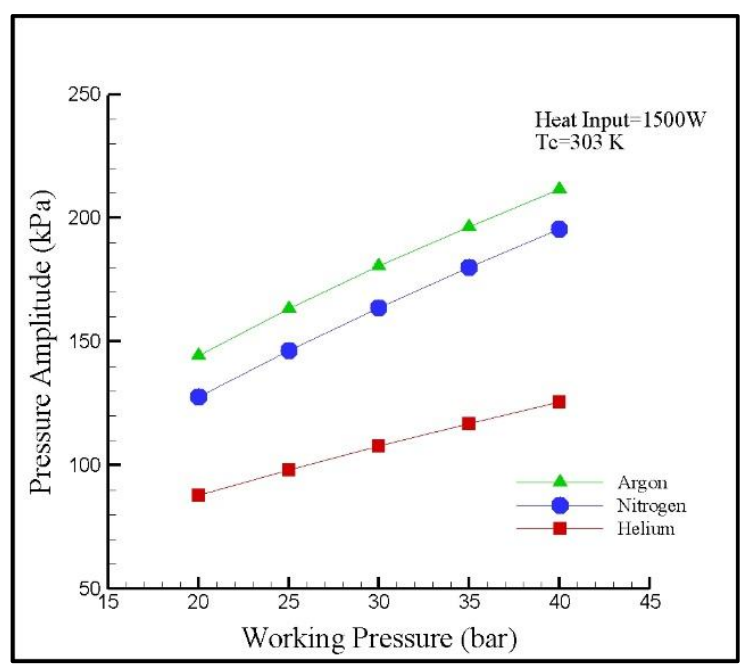

Graph- 1: Effect of working pressure on pressure amplitude

It can be seen that as the working pressure increases, the pressure amplitude increases. Also, this trend is followed by all the gases used in simulation. At any working pressure, the maximum pressure amplitude achieved is for Ar, followed by $\mathrm{N}_{2}$ and least for He. The present trend matches with the trend observed during the experiments performed by Hao et.al on standing wave thermoacoustic prime mover [6].

\subsubsection{Onset Temperature}

The Graph. 2, shows the behaviour of the onset temperature for $\mathrm{Ar}, \mathrm{He}$ and $\mathrm{N}_{2}$ as the working pressure varies. The onset temperature of any working gas can be defined as that temperature at which the thermoacoustic phenomenon begins in the prime mover.

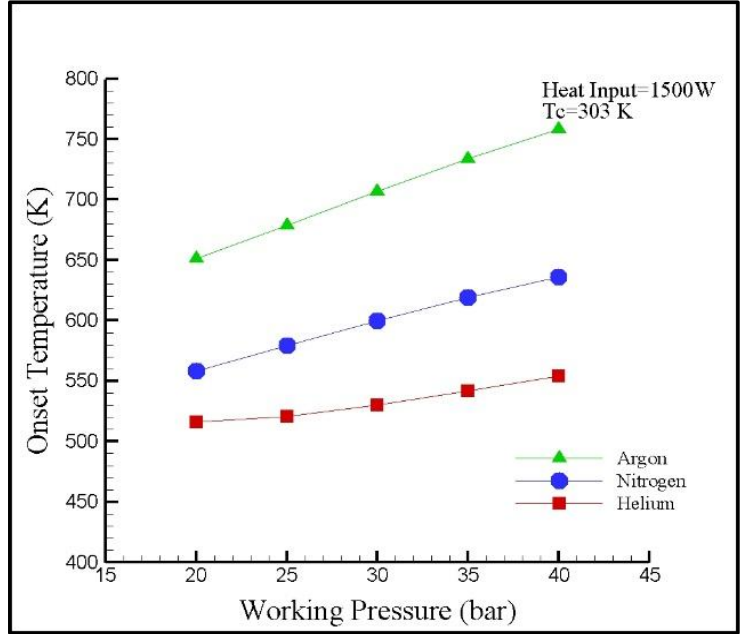

Graph-2: Effect of working pressure on onset temperature

The graph depicts that as the working pressure increases, the onset temperature of the gases increases. This pattern is followed by all the three gases. Maximum onset temperature is for Ar, followed by $\mathrm{N}_{2}$ and minimum for He. Similar comparison was observed by Hao et.al [6]

\subsubsection{Resonant Frequency}

Graph. 3 shows the variation of the resonant frequency with respect to working pressure.

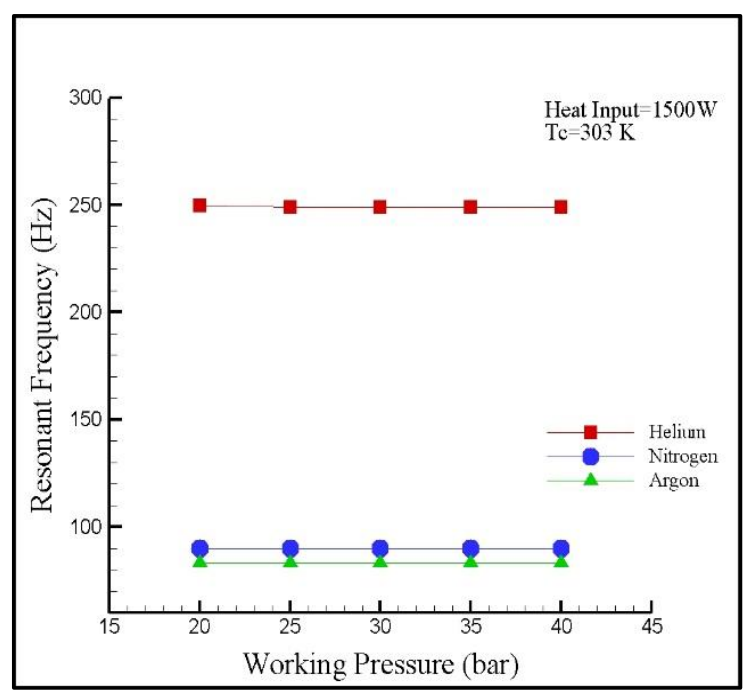

Graph- 3: Effect of working pressure on resonant frequency

It is seen from the graph that there is negligible effect of the working pressure on resonant frequency. The same trend is followed for $\mathrm{He}, \mathrm{N}_{2}$ and $\mathrm{Ar}$ gases, among which helium has the highest value, while nitrogen and argon has almost same value. The trend observed through simulation is similar to the trend obtained by Hao et.al during the experiments [6]. The 
main reason behind this behaviour is that the resonant frequency depends on the type of the gas and geometry of the prime mover and not on the working pressure [5].

\subsection{Effect of Heat Input}

In addition to the working pressure, variation in heat input to the prime mover also has a significant effect on its performance. Throughout the simulation, He was used as the working gas and the temperature of cold heat exchanger was maintained at a constant temperature of $303 \mathrm{~K}$.

\subsubsection{Pressure Amplitude}

The Graph. 4 shows the variation of pressure amplitude at different working pressures as the heat input to the system varies.

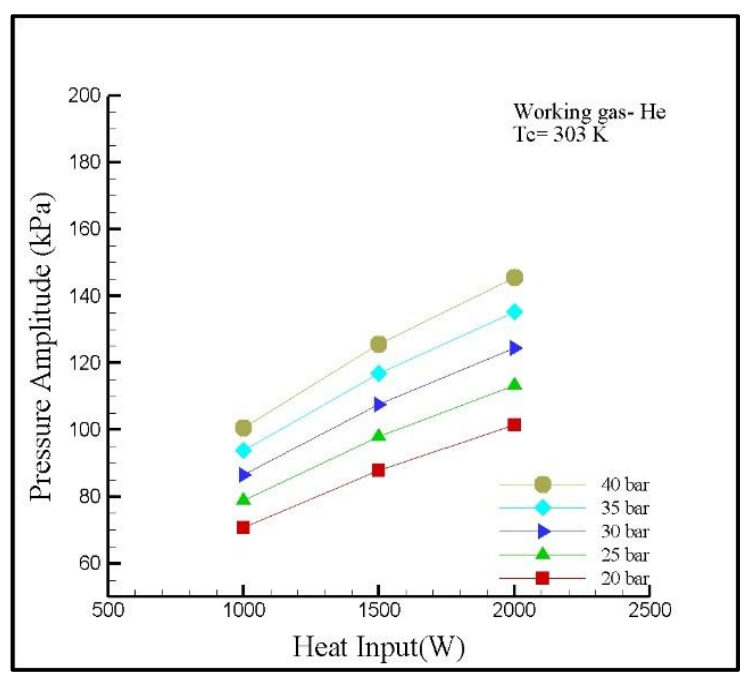

Graph- 4: Effect of heat input on pressure amplitude

From the graph, it can be seen that as the heat input increases, the pressure amplitude increases significantly. This trend is followed at all working pressures. Chen et.al observed similar trend in his work on thermoacoustic engines [7]. This is obvious as more heat energy is available for conversion into acoustic power.

\subsubsection{Onset Temperature}

Graph 4 shows the variation of onset temperature with respect to heat input at different working pressures.

It can be seen from the graph that as the heat input increases, the onset temperature increases gradually. At all working pressures, the same behaviour of onset temperature was observed. In other words it can be said that higher rate of heat transfer leads to higher onset temperature [5].

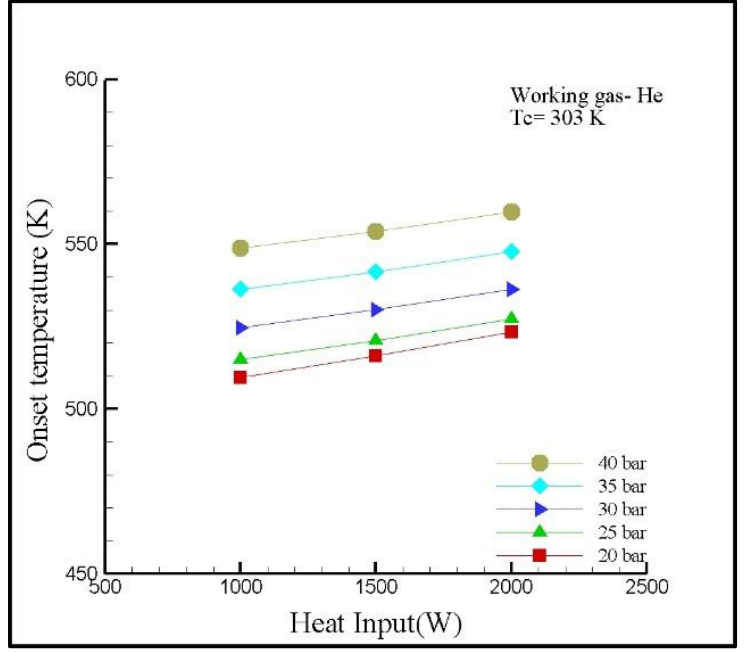

Graph- 5: Effect of heat input on onset temperature

\subsubsection{Resonant Frequency}

The effect of heat input on resonant frequency is shown in Graph. 6.

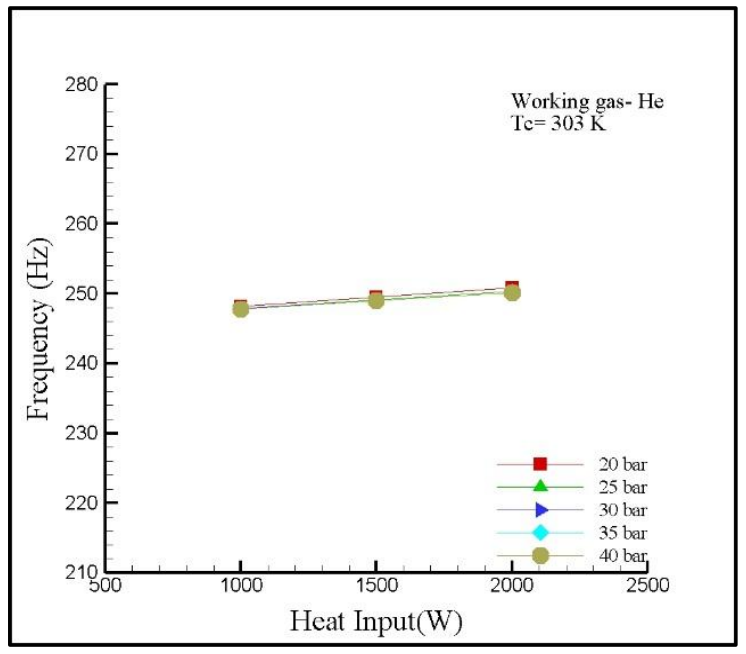

Graph- 6: Effect of heat input on resonant frequency

The graph depicts that the variation in heat input has negligible effect on the frequency. And also same trend was observed at all the working pressures.

\subsection{Effect of He-Ar Mixture}

Most of the times, it is preferred to use mixture of various gases as the working fluid in the prime mover. It helps to utilize the beneficial properties of both the gases. In this simulation, binary mixture of He-Ar was used to evaluate the performance of the engine in terms of pressure amplitude, onset temperature and frequency. 


\subsubsection{Pressure Amplitude}

Graph. 6 shows the impact of mole fraction of Helium in HeAr mixture on the pressure amplitude at different working pressure.

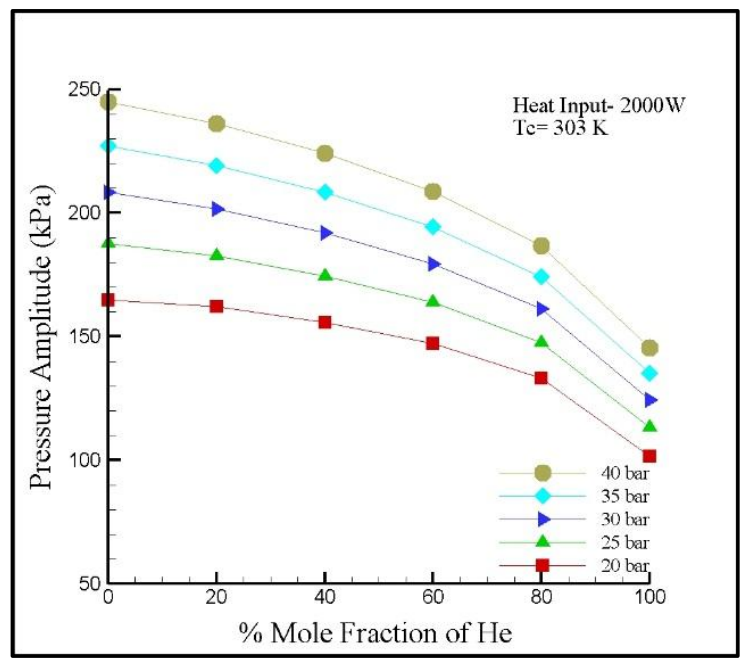

Graph- 7: Effect of mole fraction of He on pressure amplitude

As the mole fraction of $\mathrm{He}$ in the mixture goes on increasing, the pressure amplitude of the engine goes on reducing [6]. This means that more the amount of Argon in the mixture more will be the pressure amplitude. Similar trend is observed at all working pressures.

\subsubsection{Onset Temperature}

The Graph. 8 shows the variation of onset temperature as the mole fraction of $\mathrm{He}$ in the mixture increases.

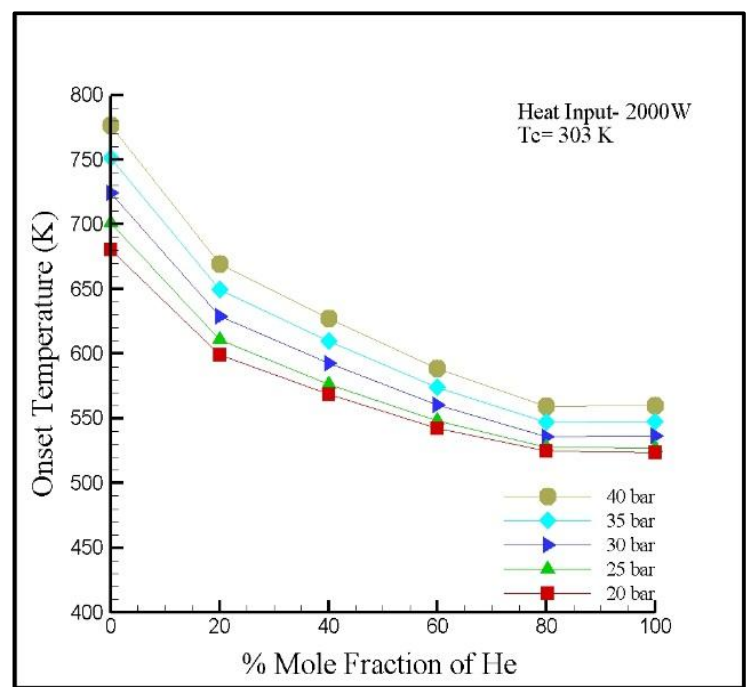

Graph- 8: Effect of mole fraction of He on onset temperature
It can be seen that as the mole fraction of $\mathrm{He}$ in the mixture increases, the onset temperature keeps on reducing [6]. The change in onset temperature is significant in the range of $0 \%$ $60 \%$ mole fraction of He. The same pattern is observed at different working pressures.

\subsubsection{Resonant Frequency}

As shown in Graph. 9, the effect of mole fraction of He on the resonant frequency has been plotted.

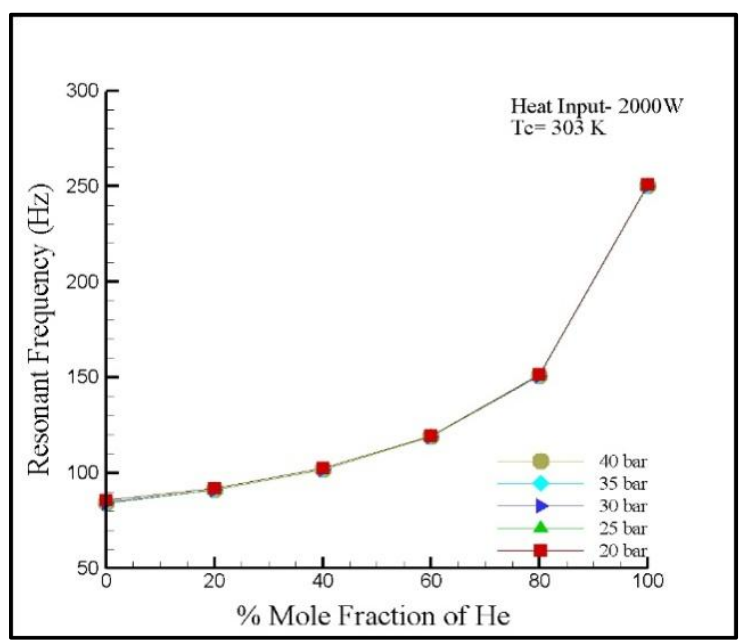

Graph- 8: Effect of mole fraction of He on resonant Frequency

Graph shows that the resonant frequency increases as the mole fraction of $\mathrm{He}$ increases. It also shows that the resonant frequency is independent of working pressure.

\section{CONCLUSIONS}

In the present work of simulation using DeltaEC, it can be concluded that Argon has the maximum pressure amplitude at all working pressures, while Helium has the minimum pressure amplitude. But, in case of Argon the onset temperature is high as compared to Helium and Nitrogen. So, it becomes unfavourable to use Argon as working gas in the prime mover when a low grade form of heat energy is used as a source of heat to the prime mover. Therefore, to get a balance between onset temperature and pressure amplitude, a mixture of He-Ar can be used in appropriate composition. Graphs show that a mixture in the range of $40 \%-60 \%$ mole fraction of He has the lowest onset temperature, which states that it is the most suitable composition of He-Ar mixture for low grade supply of energy such as solar energy, biomass and waste heat exhaust from internal combustion engine [8]. Resonant frequency depends only on the working gas and is completely independent of working pressure and heat input to the prime mover. 
For $\mathrm{He}, \mathrm{Ar}$ and $\mathrm{N} 2$, both pressure amplitude and onset temperature increases with increase in working pressure and heat input. To put up in a nut shell, depending on the required output and grade of heat energy available, the working gas should be selected wisely.

\section{REFERENCES}

[1] Mehta SM, Desai KP, Naik HB, Atrey MD, "Design of Standing Wave Type Thermoacoustic Prime Mover for $300 \mathrm{~Hz}$ Operating Frequency", 2012, PhD Thesis, SVNIT, INDIA.

[2] Ishikawa H, Hobson PA, "Optimization of heat exchanger design in a thermoacoustic engine using a second law analysis", International Community Heat and Mass Transfer 1996, pp. 325-34

[3] Swift GW, "Thermoacoustic: A Unifying perspective for some engines and refrigerators", $5^{\text {th }}$ Edition, New York, Melville, Los Alamos National Laboratory, 2001.

[4] Hariharan NM, Sivashanmugam P, Kasthurirengan S, "Effect of resonator length and working fluid on the performance of twin thermoacoustic heat engineExperimental and simulation studies", Computers and fluids, Vol. 75, 2013, pp.51-55.

[5] Shuliang Zhou, Yoichi Matsubara, "Experimental research of thermoacoustic prime mover", Cryogenics 387, 1998, pp.813-822.

[6] Hao XH, Ju YL, Upendra Behera, Kasthurirengan S, "Influence of working fluid on the performance of a standing wave thermoacoustic prime mover", Cryogenics 51, 2011, pp.559-561.

[7] Tang K, Chen GB, Jin T, Bao R, Li XM, "Performance comparison of thermoacoustic engines with constant diameter resonant tube and tapered resonant tube", Cryogenics 46, 2006, pp.699-704.

[8] Gardner DL, Howard CQ, "Waste heat driven thermoacoustic engine and refrigerator", Proceedings of Acoustics, Adelaide, Australia 2009.

\section{BIOGRAPHIES}

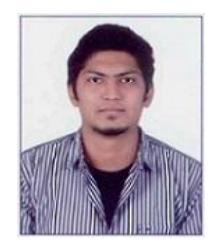

Name: Ajinkya Sarode, Currently pursuing M.Tech in SVNIT, Surat, Gujarat (INDIA) in the field of Thermal System Design. My area of research is thermoacoustic, heat transfer and renewable energy resources.

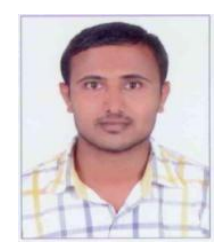

Name: Vijay Dighe, Currently pursuing M.Tech in SVNIT, Surat, Gujarat (INDIA) in the field of Thermal System Design. Research area is heat transfer and thermoacoustic.

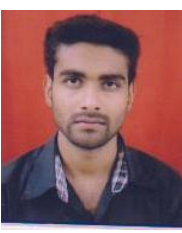

Name: Vinay Ingle, Currently pursuing M.Tech in SVNIT, Surat, Gujarat (INDIA) in the field of Thermal System Design. I am interested in thermoacoustic and cryocoolers.

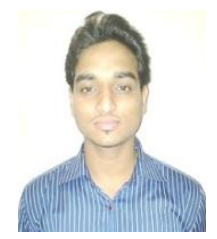

Name: Nilesh Kolekar, Currently pursuing M.Tech in SVNIT, Surat, Gujarat (INDIA) in the field of Thermal System Design. Thermoacoustics and refrigeration are my key areas of interest. 